Bitte beachten Sie: Aufgrund der Verbreitung des Coronavirus werden Veranstaltungen verschoben oder fallen kurzfristig aus. Bitte prüfen Sie vorher, ob ein Kongress stattfindet. \author{
08. - 11. September 2021 \\ Hamburg, Deutschland
}

31. Deutscher Hautkrebskongress / ADO-Jahrestagung

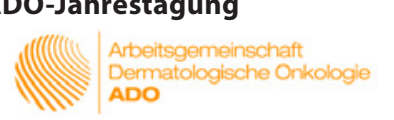

16. Deutscher Allergiekongress 30. September - 02. Oktober 2021 Dresden, Deutschland

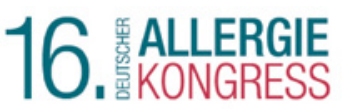

Jahrestagung der Österreichischen Gesellschaft für Dermatologie und Venerologie (ÖGDV)

25. - 27. November 2021

Salzburg, Österreich
Sa.

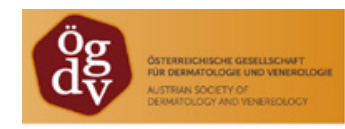

\section{JULI 2021}

38. Erlanger Minisymposium «Allergologie - Neues aus Forschung und Klinik»

24. Juli 2021

Erlangen, Deutschland, und Virtuell

AUGUST 2021

1st ADCARE Conference 2021

12. - 13. August 2021

Berlin, Deutschland

GA²LEN ADCARE - Atopic Dermatitis Centres of Reference and Excellence (ga2len-adcare.net)

Düsseldorfer Allergologie- und Immunologietagung

20. - 21. August 2021

Düsseldorf, Deutschland

Hautkrebs- und Systemtherapiesymposium Rhein-Ruhr

20. - 21. August 2021

Köln, Deutschland

103. Jahresversammlung der Schweizerischen Gesellschaft für Dermatologie und Venerologie

25. - 27. August 2021

Genf, Schweiz

https://www.sgdv-congress.ch/de/

\section{Allergologie Kompakt}

27. - 28. August 2021

Allergieakademie der DGAKI

Virtuell

92. Jahrestagung der Norddeutschen

Dermatologen

27. - 29. August 2021

Rostock, Deutschland

14. S-thetic-Circle

27. - 28. August 2021

Düsseldorf, Deutschland
10. Tagung der DWFA Sommerakademie 28. - 29. August 2021

Köln Deutschland

\section{SEPTEMBER 2021}

Haut-Update Spezial «Real-World-Daten Psoriasis - Meet the Experts» 01. September 2021 Virtuell

35. Jahrestagung Deutsche Gesellschaft für Dermatochirurgie

03. - 05. September 2021

Hamburg, Deutschland

63. Jahrestagung der Deutschen Gesellschaft für Phlebologie 08. - 11. September 2021

Aachen, Deutschland

Allergie im Kloster 2021

10. - 11. September 2021

Eltville, Deutschland

4. Leipziger Allergiegespräch
11. September 2021
Leipzig, Deutschland

16. Tagung der Arbeitsgemeinschaft für Berufs- und Umweltdermatologie 16. - 18. September 2021

Dresden, Deutschland

12. Herbstsymposium der Hautklinik Nürnberg

18. September 2021

Nürnberg, Deutschland

27. APD Jahrestagung 2021 Hamburg

18. September 2021

https://www.akpsychderm.de/

Virtuell
28. Jahrestagung der Arbeitsgemeinschaft Dermatologische Histologie (ADH)

24. - 25. September 2021

https://www.adh-kongress.de/

Virtuell

excellence in oncology - Hauttumoren: NMSC - Kutane Lymphome - Melanom 25. September 2021

Düsseldorf, Deutschland

3. Ulmer Dermatologie für die Arztpraxis 25. September 2021

Ulm, Deutschland

55. Wissenschaftliche Tagung der Deutschsprachigen Mykologischen Gesellschaft e.V.

27. - 29. September 2021

Erlangen, Deutschland

https://www.dmykg-kongress.de/

\section{0th EADV Congress}

29. September - 02. Oktober 2021

Wien, Österreich

\section{OKTOBER 2021}

30. Jahrestagung der Deutschen Dermatologischen Lasergesellschaft 01. - 03. Oktober 2021

Stuttgart, Deutschland

\section{NOVEMBER 2021}

DDA-Zertifizierungskurs

Teledermatologie

20. November 2021

Berlin, Deutschland

https://akademie-dda.de/

zertifikate/\#c10000580

information@karger.com 